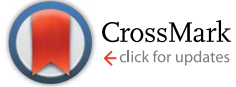

Cite this: DOI: $10.1039 / \mathrm{c} 4 \mathrm{sm} 00891 \mathrm{j}$

Received 24th April 2014 Accepted 7th July 2014

DOI: $10.1039 / c 4 s m 00891 j$

www.rsc.org/softmatter

\section{Elastocapillary deformations on partially-wetting substrates: rival contact-line models $\uparrow$}

\author{
Joshua B. Bostwick, ${ }^{* a}$ Michael Shearer ${ }^{\mathrm{b}}$ and Karen E. Daniels ${ }^{\mathrm{c}}$
}

A partially-wetting liquid can deform the underlying elastic substrate upon which it rests. This situation requires the development of theoretical models to describe the wetting forces imparted by the drop onto the solid substrate, particularly those at the contact-line. We construct a general solution using a displacement potential function for the elastic deformations within a finite elastic substrate associated with these wetting forces, and compare the results for several different contact-line models. Our work incorporates internal contributions to the surface stress from both liquid/solid $\Sigma_{\text {ls }}$ and $\Sigma_{\text {sg }}$ solid/gas solid surface tensions (surface stress), which results in a non-standard boundary-value problem that we solve using a dual integral equation. We compare our results to relevant experiments and conclude that the generalization of solid surface tension $\Sigma_{\mathrm{ls}} \neq \Sigma_{\mathrm{sg}}$ is an essential feature in any model of partial-wetting. The comparisons also allow us to systematically eliminate some proposed contact-line models.

\section{Introduction}

The deformation induced by a drop of liquid resting on an elastic substrate has been studied for some time. ${ }^{1-3}$ Describing such deformations has led to the development of the field of elastocapillarity, in which elastic stresses are coupled to surface tension (capillary forces). Among the many biological, medical and industrial applications that involve the interaction of soft substrates with fluid interfaces ${ }^{4}$ are enhanced condensation on soft substrates ${ }^{5}$ and adhesion by liquid bridges. ${ }^{6}$ Despite much progress motivated by specific applications, a fundamental characterization of how a liquid wets a soft elastic solid remains elusive.

In problems coupling elasticity to capillarity, the wetting properties of the substrate strongly control the material response. For a liquid on a hard substrate, these wetting properties are defined by the Young-Dupré equation,,$^{7,8}$

$$
\sigma_{\mathrm{sg}}-\sigma_{\mathrm{ls}}=\sigma \cos \alpha,
$$

which relates the liquid/gas $\sigma$, liquid/solid $\sigma_{\text {ls }}$ and solid/gas $\sigma_{\text {sg }}$ surface tensions to the static contact-angle $\alpha$. Fig. 1 illustrates the interpretation of the Young-Dupré relationship as a horizontal force balance. Note that this formulation leads to an imbalance of forces normal to the solid substrate with

\footnotetext{
${ }^{a}$ Department of Engineering Science and Applied Mathematics, Northwestern University, Evanston, IL 60208, USA.E-mail: joshua.bostwick@northwestern.edu ${ }^{b}$ Department of Mathematics, North Carolina State University, Raleigh, NC 27695, USA

${ }^{c}$ Department of Physics, North Carolina State University, Raleigh, NC 27695, USA $\dagger$ Electronic supplementary information (ESI) available. See DOI: $10.1039 / \mathrm{c} 4 \mathrm{ra03286a}$
}

magnitude $F_{\perp}^{\mathrm{CL}}=\sigma \sin \alpha$. The classical model of wetting of soft substrates includes this normal contact-line force applied as a point load at the contact-line, as well as the capillary pressure $p=2 \sigma \sin \alpha / R$ uniformly distributed along the liquid/solid surface area, as shown in Fig. 2. More recently, alternative models of wetting have been proposed to properly account for intrinsic surface stresses in the elastic substrate and to distinguish surface stress from surface energy. ${ }^{\mathbf{9 1 0}}$ For these models, thermodynamics dictates that the surface stress $\Sigma$ is related to the surface energy $\sigma$ by the Shuttleworth equation, $\Sigma_{\mathrm{AB}}=\sigma_{\mathrm{AB}}+$ $\partial \sigma_{\mathrm{AB}} / \partial \varepsilon$ with $\varepsilon$ the bulk strain parallel to the interface, reflecting an energetic penalty for deformation. ${ }^{\mathbf{1 1}}$ Here A, B represent the phases on either side of the interface. For substrates with an incompressible surface layer, the surface stress $\Sigma$ is equal to the surface energy $\sigma$ and both can be referred to as surface tension (Weijs et al. ${ }^{\mathbf{1 0}}$ ). Herein, we refer to $\sigma_{\mathrm{AB}}$ as the surface tension and $\Sigma_{\mathrm{AB}}$ as the solid surface tension. The result of the new models is to augment the classical model with a contact-line force $F_{\|}^{\mathrm{CL}}$ parallel to the solid and directed into the liquid phase.

In this paper we formulate a general model that describes the deformations of an elastic substrate by a partially-wetting liquid drop. The general model, formulated in terms of a displacement potential function, accommodates three rival

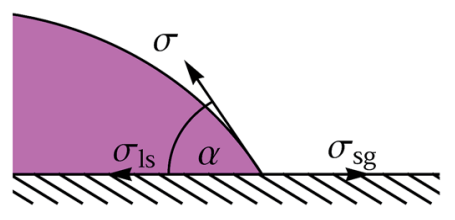

Fig. 1 The Young-Dupré eqn (1) schematically as a horizontal force balance. 

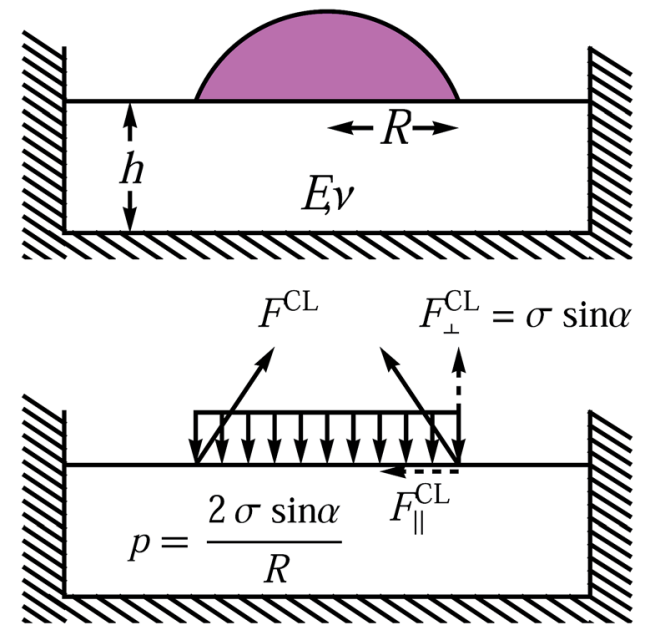

Fig. 2 Definition sketch: a liquid droplet with contact-line radius $R$ wetting an elastic substrate of height $h$, elastic modulus $E$ and Poisson ratio $\nu$ and the associated wetting forces included in the model; the capillary pressure $p$ and contact-line force $F^{\mathrm{CL}}$.

contact-line models. By introducing both liquid/solid $\Sigma_{1 \mathrm{~s}}$ and solid/gas $\Sigma_{\text {sg }}$ solid surface tensions, we generalize the work of Style and Dufresne ${ }^{12}$ on neutrally-wetting substrates $\left(\alpha=90^{\circ}\right)$ to partially-wetting substrates $\left(\alpha \neq 90^{\circ}\right)$. This leads to a force boundary condition at the substrate surface that varies along the problem domain. We construct a solution to this nonstandard problem by setting up a dual integral equation that results from extending the boundary condition into the complementary interval. We compare computed displacement fields from the general model to experimental results. The markedly different displacement fields predicted by the different models eliminates one model, and suggests suitable experiments to further resolve which of the others are most plausible.

Elastocapillary phenomena generally become important when the liquid surface tension $\sigma$ and the elastic resistance of the solid substrate have similar magnitude, as measured by the elastocapillary number $r=\sigma / E L$. Here $E$ is the elastic modulus of the substrate and $L$ is a characteristic length scale. For most liquids of interest $\sigma=10-100 \mathrm{mN} \mathrm{m}^{-1}$, and to adjust $Y$ it is typically easier to change $L$ or $E$. Experiments on the wrinkling of elastic sheets ${ }^{\mathbf{1 3 - 1 6}}$ and capillary origami ${ }^{17}$ use small $L$. Using silicone gel, ${ }^{18,19}$ gelatin $^{20,21}$ or agar gel ${ }^{22}$ as a solid substrate allows $E$ to be controllably tuned over several orders of magnitude. In systems without an intrinsic length scale, the elastocapillary length $\ell=\sigma / E$ sets the size of the elastic deformation. For reference, water $\left(\sigma=72 \mathrm{mN} \mathrm{m}^{-1}\right)$ on a silicone gel substrate $\left(E=3 \mathrm{kPa}\right.$ ) yields deformations of order $\ell \sim 10^{-6} \mathrm{~m}$. This is distinct from thin solids, such as those utilized in capillary origami experiments, where an alternative elastocapillary length $\sqrt{B / \sigma}$ can be defined using the bending modulus $B$ as a measure of the elastic resistance.

Many of the relevant experimental studies mentioned above involve neutrally-wetting $\left(\alpha=90^{\circ}\right)$ substrates. ${ }^{13,18}$ Studies of partial-wetting generally involve adding surfactant to the liquid to adjust the liquid/gas surface tension. Schroll et al. ${ }^{16}$ study how the wrinkling of ultra-thin elastic sheets due to a droplet is affected by the presence of a liquid bath covered in a predetermined surfactant concentration. They derive nearthreshold and far-from-threshold limits that recover experimental observations. Daniels et al. ${ }^{22}$ have shown that a droplet of surfactant-laden liquid placed on an agar gel can fracture the substrate in a starburst pattern. The number of arms in a given starburst is controlled by the surface tension contrast $\sigma_{\mathrm{sg}}-\sigma$, an alternative measure of the degree to which a liquid partially wets a soft solid. Bostwick and Daniels ${ }^{23}$ developed a model to predict the number of arms for this situation, and have shown that the location of the contact-line, which depends upon $\alpha$ and the droplet volume $V$, is the critical parameter in wavenumber selection, in agreement with experiments. Lastly, Style et al. ${ }^{24}$ study the contact mechanics of glass particles pressed into soft materials. These results show that $\alpha$ obeys a generalized YoungDupré equation when the indenting particle size is on the order of the elastocapillary length $\ell$.

Theory of the spreading of liquids over compliant substrates naturally relies upon an appropriate characterization of the physics of wetting, in much the same way that traditional dynamic spreading laws for liquids on rigid solids ${ }^{25-27}$ build upon the static Young-Dupré equation. For a liquid spreading on a soft viscoelastic substrate, Kajiya et al. ${ }^{28}$ show that the liquid can move continuously or with stick-slip motion depending upon the ratio of the loss to storage modulus. ${ }^{29}$ The motion of a liquid on a soft substrate experiences viscoelastic braking from the wetting ridge at the contact-line..$^{30-32}$

Hence, a description of the deformation field is needed to study the dynamics of spreading. Most of the existing theoretical models are only valid for neutrally-wetting $\left(\alpha=90^{\circ}\right)$ substrates $^{12,18}$ or straight 2D contact-lines. ${ }^{33}$ Alternative methods employ computational approaches such as density functional theory (DFT) ${ }^{34}$ and molecular dynamic (MD) simulations $\mathrm{s}^{35}$ to gain a more thorough understanding of the wetting forces acting at the contact-line. The thrust of our work lies in the modeling of partially-wetting systems.

We begin by formulating a mathematical model (Section 2) for the deformation of an elastic substrate due to a partiallywetting liquid droplet. The effects of partial-wetting appear in (i) the contact-line force boundary conditions and (ii) the solid surface tensions $\Sigma_{\mathrm{sg}} \neq \Sigma_{\mathrm{ls}}$. Three rival contact-line models are introduced and the governing equations are recast using a displacement potential. We then construct a general solution of the dual integral equation that results from the discontinuity in solid surface tension $\Sigma$ along the surface of the substrate. The discontinuity occurs where the interface changes from liquid/solid to solid/gas. Our numerical results are presented in Section 3, where we contrast measures of the elastic displacement field for the different models as they depend upon the model parameters. Comparisons between the predicted fields and relevant experiments allow us to systematically eliminate some proposed contact-line models. In addition, we show that the generalization of solid surface tension is an important feature for modeling wetting on soft substrates. We conclude with some remarks in Section 4 on 
future studies that could help resolve the issue of which model of wetting is most realistic.

\section{Mathematical formulation}

A partially-wetting droplet resting on a solid substrate is held by liquid-gas surface tension $\sigma$ at its free surface. For negligible gravitational forces, the equilibrium shape is a spherical-cap with contact-line radius $R$, static contact-angle $\alpha$ and volume

$$
\frac{V}{R^{3}}=\frac{\pi}{3} \frac{\left(2-3 \cos \alpha+\cos ^{3} \alpha\right)}{\sin ^{3} \alpha} .
$$

Note that for fixed volume drops, $R$ and $\alpha$ are not independent parameters. The linear elastic substrate has thickness $h$ and is characterized by an elastic modulus $E$ and Poisson ratio $\nu$, as shown in Fig. 2. The liquid interacts with the solid through both the capillary pressure $p=2 \sigma \sin \alpha / R$ uniformly distributed over the liquid/solid contact area and the unbalanced contactline force $F^{\mathrm{CL}}$ applied at the contact-line radius $R$ ( $c f$. Fig. 2). We compute the elastic response in the substrate due to these wetting forces.

\subsection{Field equations}

We begin by introducing the axisymmetric displacement field $u$,

$$
u=u_{r}(r, z) \hat{e}_{r}+u_{\mathrm{z}}(r, z) \hat{e}_{z}
$$

in cylindrical coordinates $(r, z)$, which satisfies the governing elastostatic Navier equations,

$$
(1-2 \nu) \nabla^{2} u+\nabla(\nabla \cdot u)=0 .
$$

The strain field $\varepsilon$ is defined as

$$
\varepsilon=\frac{1}{2}\left(\nabla u+(\nabla u)^{t}\right)
$$

while the stress field $\tau_{i j}$ for this linear elastic solid is given by

$$
\tau_{i j}=\frac{E}{1+v}\left(\varepsilon_{i j}+\frac{v}{1-2 v} \varepsilon_{k k}\right) .
$$

\subsection{Boundary conditions}

We assume the elastic substrate is pinned to a rigid support at $z$ $=0$ by enforcing a zero displacement boundary condition there,

$$
u(r, 0)=0 .
$$

On the free surface $z=h$, we specify the surface tractions

$$
\begin{array}{cc}
\tau_{r z}(r, h)=F_{r}(r), & 0 \leq r \leq \infty, \\
\tau_{z z}(r, h)-\Sigma_{\mathrm{ls}} \nabla_{\|}^{2} u_{z}(r, h)=F_{z}(r), & 0 \leq r \leq R, \\
\tau_{z z}(r, h)-\Sigma_{\mathrm{sg}} \nabla_{\|}^{2} u_{z}(r, h)=F_{z}(r), & R<r \leq \infty .
\end{array}
$$

here $\nabla_{\|}^{2}$ is the surface Laplacian and $F_{z}(r)$ and $F_{r}(r)$ are the applied vertical and horizontal forces associated with the liquid/solid interactions. These forces are model-dependent, and their particular choice will be discussed in Section 2.3. As discussed by Style and Dufresne, ${ }^{12}$ Jerison et al.,${ }^{18}$ introducing the $\Sigma$ solid surface tension (i) allows for the modeling of neutrally-wetting substrates $\Sigma_{\text {sg }}=\Sigma_{\text {ls }}\left(\alpha=90^{\circ}\right)$ and (ii) regularizes the singularity associated with applying a $\delta$-function force to the medium's surface. Here, we extend this technique to allow us to model partially-wetting substrates with $\Sigma_{\mathrm{sg}} \neq \Sigma_{\mathrm{ls}}$ corresponding to $\alpha \neq 90^{\circ}$.

\subsection{Wetting forces}

We now develop a model for the forces $F_{z}, F_{r}$ associated with the wetting of a liquid droplet on a soft elastic substrate. For a liquid droplet held by uniform surface tension $\sigma$, the vertical wetting forces are given by

$$
F_{z}(r)=\sigma \sin \alpha\left(\delta(r-R)-\frac{2}{R} H(R-r)\right) .
$$

here the capillary pressure $p=2 \sigma \sin \alpha / R$ (second term) is uniformly distributed over the liquid/solid surface area by the Heaviside function $H(R-r)$, whereas the unbalanced vertical contact-line force $F_{z}^{\mathrm{CL}}=\sigma \sin \alpha$ (first term) is applied as a point load using a delta function $\delta(r-R)$ at the contact-line $r=R$. Note the orientation of the applied forces; the capillary pressure $p$ compresses the substrate, while the contact-line force $F_{z}^{\mathrm{CL}}$ tends to pull the substrate upwards. In fact, the upward contactline force precisely balances the net downward force from the pressure. Eqn (9) is the standard, or classic, description of wetting of soft substrates.

More recent models of wetting have introduced an uncompensated parallel contact-line force $F_{r}(r)$, in addition to the vertical wetting forces (9) described above. ${ }^{\mathbf{1 0 , 3 4}}$ Here we would like to construct a general solution for the models of wetting discussed below in order to contrast the resulting elastic fields. Each model for the uncompensated parallel contact-line force can be written as

$$
F_{r}(r)=F_{r}^{\mathrm{CL}} \delta(r-R)
$$

with the coefficient $F_{r}^{\mathrm{CL}}$ for the respective model shown in Table 1 .

\subsection{Summary of wetting models}

Model I corresponds to the classic picture of wetting in which the contact line exerts no horizontal force on the substrate. In contrast, Models II and III take the same form with respect to $\alpha$,

Table 1 Horizontal contact-line force $F_{r}^{\mathrm{CL}}$ for the classic description of wetting (I), used by Jerison et al., ${ }^{18}$ Style and Dufresne, ${ }^{12}$ and updated Models II and III, proposed by Das et al. ${ }^{34}$ and Weijs et al. ${ }^{10}$ respectively. Here $\sigma$ is the surface tension, $\nu$ the Poisson ratio, and $\alpha$ the contact angle given by eqn (1)

\begin{tabular}{ll}
\hline Model & $F_{\mathrm{r}}^{\mathrm{CL}}$ \\
\hline I & 0 \\
II & $-\sigma(1+\cos \alpha)$ \\
III & $-\sigma(1+\cos \alpha)\left(\frac{1-2 \nu}{1-\nu}\right)$
\end{tabular}


but have different dependence on the Poisson ratio $\nu$ of the substrate. Note that for the unusual case of $\nu=0$, Model III reduces to Model II; however, ordinary materials do not typically reach this limit. ${ }^{36}$ The more interesting case is that of incompressible substrates, for which $\nu=1 / 2$ and Model III reduces to Model I. Many soft materials are known to be highly incompressible $^{37}$ and modern measurement techniques ${ }^{\mathbf{3 8}}$ are making it possible to obtain precise values of the deviation from $1 / 2$. In particular, the experiments of Style et al. ${ }^{24}$ report $\nu=$ 0.495 for the silicone gel to which we compare model results below. Even if Model III were the correct model, the closeness to $\nu=1 / 2$ would explain why Model I has been so successful in predicting the elastic deformations on soft substrates.

\subsection{Displacement potential-Love function}

The Navier eqn (4) are simplified by introducing the Galerkin vector $G,{ }^{39}$ defined such that

$$
u=\frac{1+\nu}{E}\left(2(1-\nu) \nabla^{2} G-\nabla(\nabla \cdot G)\right)
$$

with

$$
G=\xi(r, z) \hat{e}_{z} .
$$

Sometimes the potential $\xi$ is referred to as the Love function from classical linear elasticity. We substitute (11) into the coupled system of differential eqn (4) to show that $\xi$ satisfies the biharmonic equation

$$
\nabla^{4} \xi=0
$$

The displacement (7) and traction (8) boundary conditions can similarly be written in terms of the potential function $\xi$.

\subsection{Hankel transform}

We seek solutions to (13) for the potential function using the Hankel transform pair,

$$
\begin{aligned}
& \hat{\xi}(s, z)=\int_{0}^{\infty} r \xi(r, z) J_{0}(s r) \mathrm{d} r, \\
& \xi(r, z)=\int_{0}^{\infty} s \hat{\xi}(s, z) J_{0}(s r) \mathrm{d} s,
\end{aligned}
$$

where $J_{0}$ is the Bessel function of the first kind and $s$ is the radial wavenumber.

\subsection{Reduced equations}

We introduce the following dimensionless variables;

$$
u \equiv \tilde{u} \frac{\sigma}{E}, r \equiv \tilde{r} h, z \equiv \tilde{z} h, s \equiv \frac{\tilde{s}}{h}, R \equiv \tilde{R} h .
$$

here lengths are scaled by the thickness of the elastic substrate $h$ and elastic deformations by the elastocapillary length $\ell \equiv \sigma / E$. Herein we drop the tildes for notational simplicity. Substituting the Hankel expansion (14a) into (13) gives a reduced equation for $\hat{\xi}$,

$$
\nabla^{4} \hat{\xi}=\left(\frac{\mathrm{d}^{2}}{\mathrm{~d} z^{2}}-s^{2}\right)^{2} \hat{\xi}=0,
$$

combined with the no-displacement condition on the rigid support $z=0$,

$$
\frac{\mathrm{d} \hat{\xi}}{\mathrm{d} z}=0,(1-2 \nu) \frac{\mathrm{d}^{2} \hat{\xi}}{\mathrm{d} z^{2}}-2(1-\nu) s^{2} \hat{\xi}=0 .
$$

The general solution of (16) and (17) is given by

$$
\hat{\xi}=C\left(\cosh (s z)+\frac{s z \sinh (s z)}{2(1-2 \nu)}\right)+D(s z \cosh (s z)-\sinh (s z)),
$$

with the constants $C, D$ to be determined from the traction boundary conditions (8). Here we note that the form of (8) is not amenable to standard analysis because the vertical boundary conditions $\tau_{z z}$ change along the problem domain $r \in[0, \infty]$. We address this issue in the following section by constructing a solution to this non-standard problem using a dual integral formulation. Given the solution $\hat{\xi}$, we compute $\xi$ in real space by evaluating the inverse Hankel transform (14b). Once the potential function $\xi$ is known, the displacement $\boldsymbol{u}$, strain $\varepsilon$ and stress $\tau$ fields are obtained via substitution into (11), (5) and (6), respectively.

\subsection{Dimensionless groups}

The following dimensionless groups arise naturally from the choice of scaling (15),

$$
r \equiv \frac{\sigma}{E h}, r_{\mathrm{sg}} \equiv \frac{\Sigma_{\mathrm{sg}}}{E h}, r_{\mathrm{ls}} \equiv \frac{\Sigma_{\mathrm{ls}}}{E h}, \Lambda \equiv \frac{R}{h}
$$

Here $r, r_{\text {sg }}$ and $r_{\text {ls }}$ are the liquid/gas, solid/gas and liquid/solid elastocapillary numbers and $\Lambda$ is the aspect ratio or dimensionless contact-line radius. We also define the solid surface tension contrast $\Delta r \equiv r_{\mathrm{sg}}-r_{\mathrm{ls}}$, which can be viewed as a measure of partial wetting.

\subsection{Dual integral equation}

The vertical component $\tau_{z z}$ of the traction boundary conditions (8) changes along the problem domain depending upon whether the solid substrate interacts with the liquid droplet $(r \in[0, R])$ or the passive gas $(r \in[R, \infty])$. To specify the constants $C, D$ in our general solution (18), we recast the traction boundary conditions (8) in a form amenable to a dual integral solution,

$$
\begin{gathered}
\tau_{r z}=F_{r}(r), 0 \leq r \leq \infty \\
\tau_{z z}-\Sigma_{\mathrm{sg}} \nabla_{\|}^{2} u_{z}-F_{z}(r)=\left(\Sigma_{\mathrm{ls}}-\Sigma_{\mathrm{sg}}\right) \nabla_{\|}{ }^{2} u_{z}, 0 \leq r \leq R \\
\tau_{z z}-\Sigma_{\mathrm{sg}} \nabla_{\|}^{2} u_{z}-F_{z}(r)=0, R<r \leq \infty
\end{gathered}
$$

The vertical force balance (20b) and (20c) is then written as

$$
\int_{0}^{\infty} A(s) J_{0}(s r) \mathrm{d} s=\left\{\begin{array}{cc}
G(r) & 0 \leq r \leq R \\
0 & R<r \leq \infty
\end{array}\right.
$$


with

$$
A(s)=s\left(\hat{\tau}_{z z}+\Sigma_{\mathrm{sg}} s^{2} \hat{u}_{z}-\hat{F}_{z}\right), G(r)=\Delta \Sigma \int_{0}^{\infty} s^{3} \hat{u}_{z} J_{0}(s r) \mathrm{d} s,
$$

and $\Delta \Sigma \equiv \Sigma_{\text {sg }}-\Sigma_{\text {ls. }}$ Eqn (21) is recognized as a dual integral equation with a standard solution, ${ }^{\mathbf{4 0 , 4 1}}$

$$
A(s)=\frac{2}{\pi} \int_{0}^{R} \cos s t \int_{t}^{R} \frac{r G(r)}{\sqrt{r^{2}-t^{2}}} \mathrm{~d} r \mathrm{~d} t .
$$

Note that the solution is valid over the full domain $r \in[0, \infty]$. Substituting ((18) and (22)) into (23) yields

$$
s\left(\hat{\tau}_{z z}+\Sigma_{\mathrm{sg}} s^{2} \hat{u}_{z}-\hat{F}_{z}\right)=C A_{1}(\mathrm{~s})+D A_{2}(\mathrm{~s}),
$$

where

$$
A_{k}(s)=\Delta \Sigma \frac{2}{\pi} \int_{0}^{R} \cos s t \int_{0}^{R} \frac{r}{\sqrt{r^{2}-t^{2}}} \int_{0}^{\infty} q^{3} v_{k}(q) J_{0}(q r) \mathrm{d} q \mathrm{~d} r \mathrm{~d} t
$$

and

$$
\begin{gathered}
v_{1}(q)=-\frac{q^{3} \sinh q}{2(1-2 \nu)}, \\
v_{2}(q)=q^{2} \frac{2\left(3-10 \nu+8 \nu^{2}\right) \sinh q-2 q(1-2 \nu) \cosh q}{2(1-2 \nu)} .
\end{gathered}
$$

Eqn (24) and the Hankel-transformed horizontal force balance (20a) are a linear system of equations for $C, D$, whose solution is given in the Appendix.

\section{Results}

Our goal is to contrast the three contact-line models and the interpretation of solid surface tension for partial wetting, by comparing theoretical displacement fields to relevant experiments. Some of these comparisons can be directly evaluated using data from the literature, while others identify tests which would help design future experiments. We compute the elastic fields by substituting the coefficients $C, D$ into (12) and evaluating (14b) for the displacement potential, from which the displacements $u$, strains $\varepsilon$ and stresses $\tau$ are readily obtained. These solutions provide quantitative measures of how the elastic field, for instance the vertical contact-line displacement $u_{z}^{\mathrm{CL}}$ (peak height), varies with the model parameters.

We begin by comparing our model to the experimental results of Style et al. ${ }^{19}$ who use confocal microscopy to measure surface displacements on silicone gels from partially-wetting droplets. Their focus is in how the displacement fields vary with two length scales, the contact-line radius $R$ and substrate height $h$. Fig. 3 shows how the vertical surface displacement $u_{z}$ changes across the substrate for Model I. Material parameters for our computations are taken directly from the reported values. ${ }^{\mathbf{1 9}}$ Note that $\nu \approx 1 / 2$ for silicone gels, which implies that contactline Models I and III are equivalent for these experiments. We see that our model is able to adequately reproduce the experimental results over a range of contact-line radii, which is

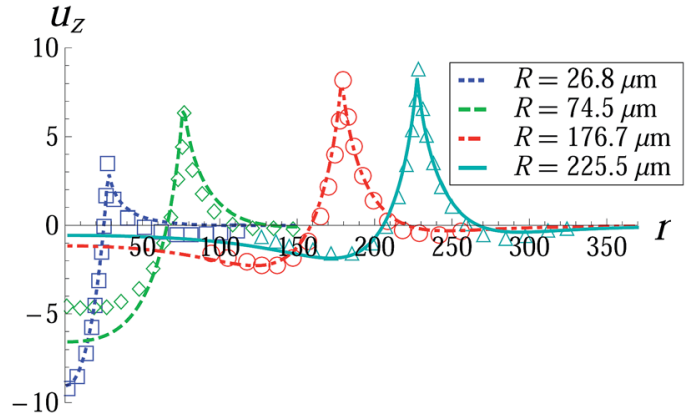

Fig. 3 Comparison with Style et al.., ${ }^{19}$ Fig. 1. Surface displacement $u_{z}$ on a $h=50 \mu \mathrm{m}$ thick substrate against $r$ from Model I, III, as it depends upon the contact-line radius $R$ for $\nu=0.5, r_{\mathrm{sg}}=0.207, \Delta r=-0.033, E$ $=3 \mathrm{kPa}, \sigma=46 \mathrm{mN} \mathrm{m}^{-1}$ and $\alpha=95^{\circ}$. Lengths are reported in $\mu \mathrm{m}$. Experimental results are shown with open symbols. Material properties are taken to be those reported in the experiments. ${ }^{19}$

achieved experimentally by varying the droplet volume while holding the other parameters fixed. The capillary pressure $p=$ $2 \sigma \sin \alpha / R$ tends to compress the material beneath the drop and is more pronounced for smaller drops, as would be expected. For larger drops $R=225.5 \mu \mathrm{m}$ (solid line type), the contact-line force dominates the elastic response and the compressive troughs on either side of the contact-line peak become nearly symmetrical, reflecting a nearly two-dimensional solution. ${ }^{\mathbf{1 8}}$ Despite the large variation in surface profile with droplet size, the local geometry of the wetting ridge remains invariant; the predicted microscopic contact angle $93.8^{\circ}$ for both contact-line Models I and II agrees well with that reported by Style et al. ${ }^{\mathbf{1 9}}$ ( $c f$. ESI, Fig. S5 $\dagger$ ). We attribute this observation to the generalization of solid surface tension.

The peak height $u_{z}^{\mathrm{CL}}$ directly at the contact-line can be used as a measure of the elastic response of the underlying substrate. In Fig. 4, we plot the peak height for Models I and III as a function of the contact-line radius $R$ for various substrate heights $h$, and compare with experiments on silicone gel substrates. ${ }^{19}$ For a fixed substrate height $h$, the peak height increases with increasing contact-line radius $R$, achieves a

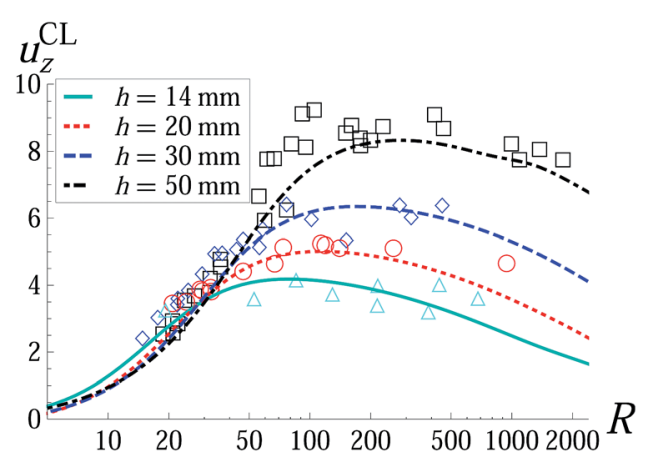

Fig. 4 Comparison with Style et al.., ${ }^{19}$ Fig. 3. Contact-line displacement $u_{z}^{\mathrm{CL}}$ computed from Model I, III against drop radius $R$, as it depends upon the substrate height $h$ for $\nu=0.5, r_{\text {sg }}=0.207, \Delta r=-0.033, E=$ $3 \mathrm{kPa}, \sigma=46 \mathrm{mN} \mathrm{m}^{-1}$ and $\alpha=95^{\circ}$. Lengths are reported in $\mu \mathrm{m}$. Experimental results are shown with open symbols. 
maximum and decreases thereafter. Smaller substrate heights lead to uniformly smaller peak heights reflecting the presence of the underlying rigid support, where the zero displacement condition (7) is enforced. In contrast, thicker substrates are less affected by the underlying support since there is more material to resist the applied surface tractions, resulting in larger peak deformations. Fig. 4 demonstrates the non-monotonic dependence on contact-line radius, consistent with experiments. We attribute this behavior to the effects of partial wetting $\left(\alpha=95^{\circ}\right)$ that result from a non-trivial difference between the liquid/solid and solid/gas solid surface tensions $\Delta r \neq 0$.

For neutrally-wetting $\alpha=90^{\circ}$ substrates $(\Delta r=0)$, the peak height is a monotonic function of the contact-line radius $\Lambda$ for Models I, III, as shown in Fig. 5. That is, the peak height increases with the contact-line radius and then plateaus. We conclude that the generalization which differentiates between the $\Sigma_{\mathrm{ls}}$ and $\Sigma_{\mathrm{sg}}$ solid surface tensions is a feature of the model that is required to reproduce the experimental data. In contrast, Fig. 5 shows that for Model II with $\Delta r=0$, the peak height is a non-monotonic function of the contact-line radius that is also consistent with experiments ( $c f$. Fig. 4).

We proceed by contrasting contact-line Models I, III and II on incompressible $(\nu=1 / 2)$ substrates. Fig. 6 plots the surface displacements $u_{z}, u_{r}$ for Models I and II on a neutrally wetting $\alpha=90^{\circ}$ substrate. The peak heights $u_{z}^{\mathrm{CL}}$ are superficially similar, but the fields vary greatly away from the contact-line. Notice that beneath the drop the field is compressive for Model I and tensile for Model II. Outside the drop, the compressive dimple is much more pronounced for Model II. Fig. 7 compares surface profiles for fluorinert drops with $\alpha=40^{\circ}$ against experiment demonstrating that Model I more accurately captures the experimental observations. Additional comparisons to experiment are given in the Supplementary Material. A more dramatic difference between Model I and II is seen in the radial surface
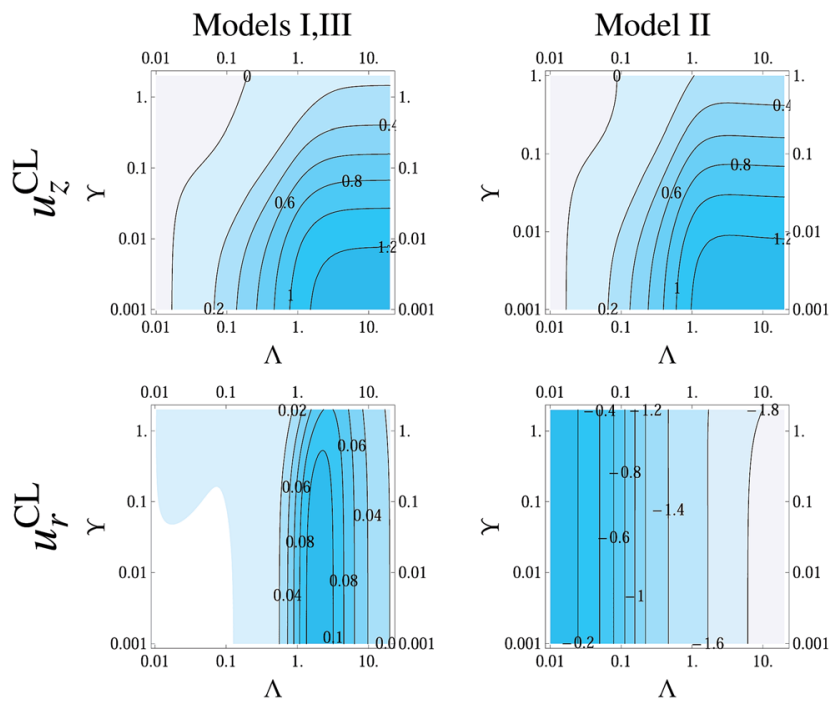

Fig. 5 Contact-line displacement comparing Models I, III and II by plotting the axial $u_{z}^{\mathrm{CL}}$ and radial $u_{r}^{\mathrm{CL}}$ displacement at the contactline $(r=\Lambda)$, as it depends upon the solid elastocapillary number $r=r_{\text {sg }}=r_{\text {ls }}$ and the contact-line radius $\Lambda$ for $\nu=1 / 2$ and $\alpha=90^{\circ}$.
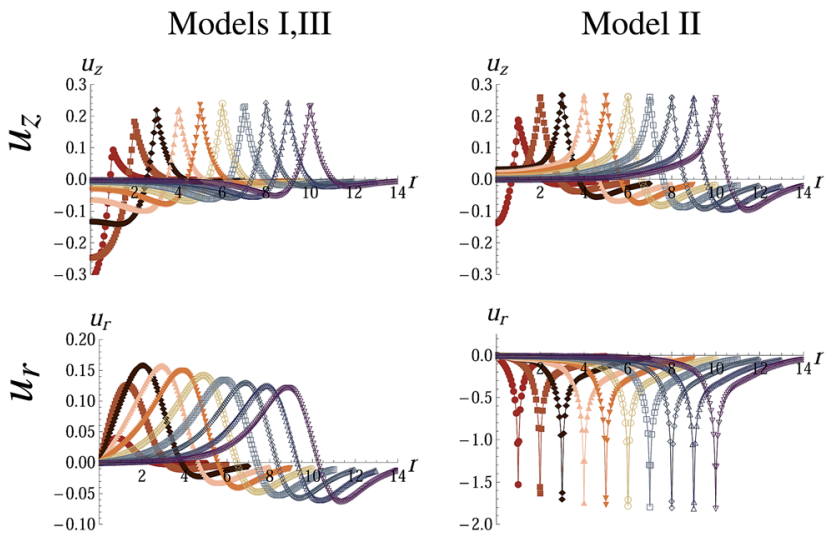

Fig. 6 Comparison between Models I, III and II by plotting the surface displacements $u_{z}, u_{r}$ on an incompressible $\nu=1 / 2$ substrate, against $r$, as it depends upon the contact-line radius $\Lambda$, for $r_{\text {ls }}=1, r_{\text {sg }}=1, \alpha=$ $90^{\circ}$. Note the different scales for the radial displacement.

displacement. For Model I, there is a peak on the droplet side and a trough on the gas side of the contact-line that eventually becomes symmetric as the contact-line radius increases. In contrast, the radial displacement is directed into the drop $\left(u_{r}<\right.$ 0 ) for Model II. In addition to the qualitative differences in the radial displacement field, note the radial displacement $u_{r}$ scale changes by an order of magnitude between Models I and II. This observation is robust and occurs over a large range of parameters $(c f$. ESI $\dagger)$. Such a dramatic effect should clearly be visible in experiment. However, Jerison et al., ${ }^{18}$ Fig. 2 measure the radial displacement field on incompressible silicone gel substrates showing a field more similar to that of Model I than Model II. We conclude that contact-line Model II does not accurately capture the existing experimental data and, hence, rule it out as a candidate contact-line law.

At this point, our candidate models have been reduced to either Model I or Model III. We have demonstrated above that the generalization of solid surface tensions $(\Delta r \neq 0)$ is an essential feature of any model. Recall that Model III includes a horizontal force that depends upon the Poisson ratio $\nu$, which

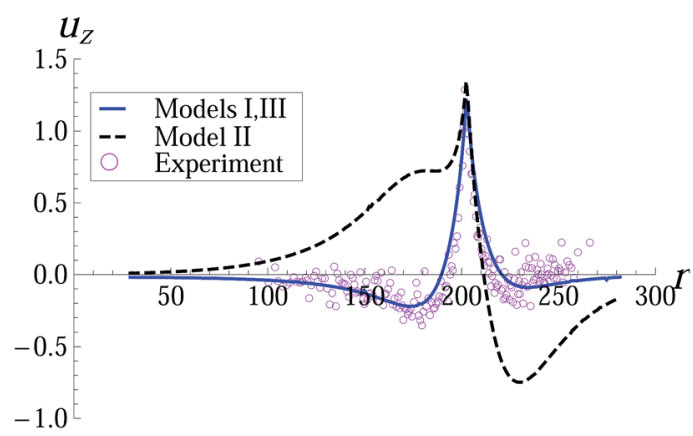

Fig. 7 Surface displacement $u_{z}$ for a fluorinert drop on a $h=23 \mu \mathrm{m}$ thick substrate against $r$ comparing Models I, III to Model II, for $R=$ $196.59 \mu \mathrm{m}, \nu=0.5, r_{\mathrm{sg}}=0.349, \Delta r=0.149, E=3 \mathrm{kPa}, \sigma=17 \mathrm{mN} \mathrm{m}^{-1}$ and $\alpha=40^{\circ}$. Experimental results are shown with open symbols. Lengths are reported in $\mu \mathrm{m}$. Material properties are taken to be those reported in the experiments. ${ }^{19}$ 

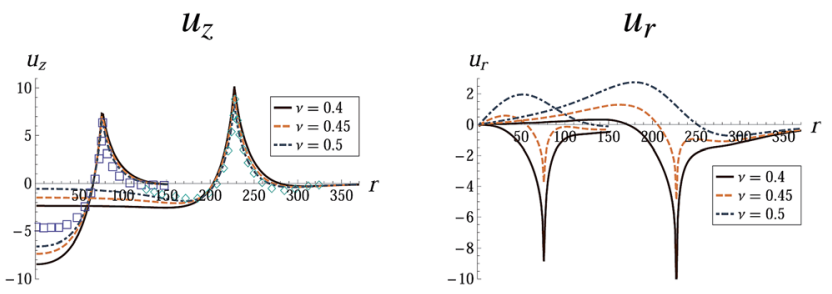

Fig. 8 Compressibility effects from contact-line Model III with $R=$ $74.5 \mu \mathrm{m}$ and $R=225.5 \mu \mathrm{m}$ : axial $u_{z}$ and radial $u_{r}$ displacement field in $\mu \mathrm{m}$, as it depends upon the Poisson ratio $\nu$, for $r_{\mathrm{sg}}=0.207, \Delta r=$ $-0.033, E=3 \mathrm{kPa}, \sigma=46 \mathrm{mN} \mathrm{m}^{-1}$ and $\alpha=95^{\circ}$. Open symbols in left sub-figure are two experiments from Style and Dufresne. ${ }^{12}$

degenerates into Model I when $\nu=1 / 2$. Fig. 8 shows the displacement fields for Model III, as they depend upon $\nu$ for nearly incompressible substrates. For the vertical displacement $u_{z}$, the peak height does not appreciably change with $\nu$, while the largest difference occurs near the center of the drop $r=0$. The most dramatic difference occurs for the radial displacement $u_{r}$, where the presence of the horizontal force dominates the elastic response, even at $\nu=0.45$ and more so as $\nu$ decreases from $1 / 2$. With regards to validation of the models, most experiments utilize nearly incompressible materials and, as we have stated, one cannot differentiate between Models I and III in this limit. Experimental measurements of the radial displacement field on compressible substrates should resolve this issue once and for all.

A typical measure of the elastic response due to a partiallywetting liquid is the contact-line displacement, which can usually be measured without sophisticated diagnostics. Another benefit is that the contact-line displacement is a scalar measure of the more complicated elastic field. Fig. 9 shows how the
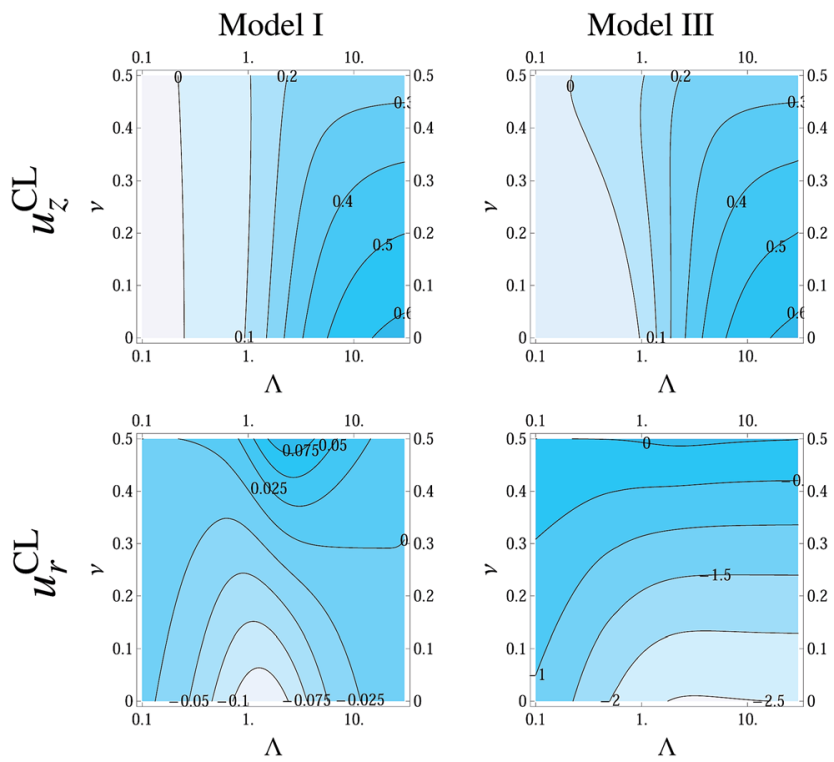

Fig. 9 Comparison of the axial $u_{z}^{C L}$ and radial $u_{r}^{C L}$ contact-line displacement for Models I and III, as it depends upon the Poisson ratio $\nu$ and the contact-line radius $\Lambda$ for $r \equiv r_{\mathrm{sg}}=r_{\mathrm{ls}}=1$ and $\alpha=90^{\circ}$.
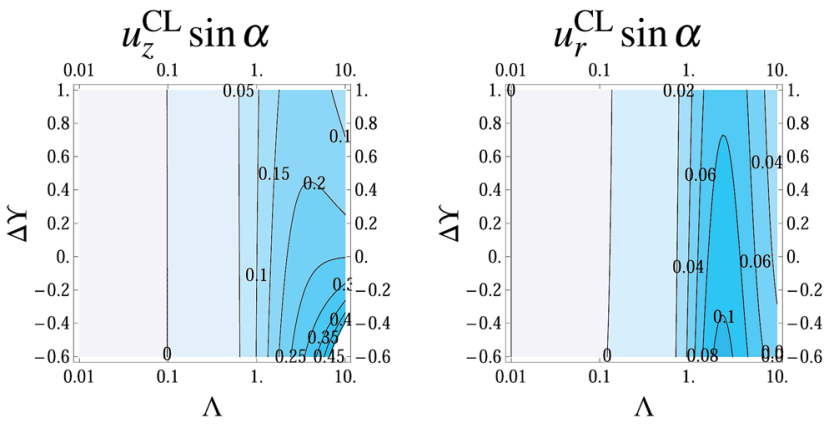

Fig. 10 Comparison of the axial $u_{z}^{\mathrm{CL}} \sin \alpha$ and radial $u_{r}^{\mathrm{CL}} \sin \alpha$ contactline displacement for Models I and III, as it depends upon $\Delta r$ and the contact-line radius $\Lambda$ for $\nu=1 / 2$ and $r_{\mathrm{sg}}=1$.

contact-line displacement for a neutrally-wetting $\left(\alpha=90^{\circ}\right)$ substrate varies with the Poisson ratio $\nu$ and solid elastocapillary number $r$ for Models I, III. The information shown here could be used in future experiments to reconcile the appropriate contact-line law, either Model I or III.

Finally, we show how the contact-line displacement varies with $\Lambda$ and $\Delta r$ in Fig. 10. Note that for partially-wetting situations, both $\alpha$ and $\Delta r$ change with the surface chemistry. Hence, we plot the displacements $u \sin \alpha$. The contact-line displacement for Model II is given in the Supplementary Material. We view Fig. 10 as a guide for future studies on partially-wetting substrates.

\section{Discussion}

We have considered the elastic deformations of a soft substrate due to the presence of a partially-wetting liquid. We construct a general solution for the displacement potential (Love function) comparing three rival contact-line models for wetting forces imparted by the liquid onto the solid. In addition, our model generalizes the concept of solid surface tension to partiallywetting substrates $\alpha \neq 90^{\circ}$, where $\Sigma_{\mathrm{ls}} \neq \Sigma_{\text {sg }}$. The result of which is a non-standard boundary-value problem that we solve using a dual integral equation. The thrust of this work is that our general solution encompasses all current contact-line models, as well as the interpretation of solid surface tension as a surface stress $\Sigma \equiv \Sigma_{\text {sg }}=\Sigma_{\text {ls }}$ or surface energy $\Sigma_{\text {sg }} \neq \Sigma_{\text {ls }}$.

We compare the computed elastic displacement fields to relevant experiments, ${ }^{18,19}$ which allows us to identify the most likely model of wetting of soft substrates from the potential candidate models. When comparing to experiment, we immediately see that the surface energy interpretation $\Delta r \neq 0$ is an essential feature that should be included in any model of partial-wetting. Contact-line Model II is ruled out as a candidate based upon the dramatic differences between the computed displacement field and experimental observations. This leaves contact-line Models I and III as possibilities. However, since the relevant experiments involve incompressible substrates $\nu \approx 1 / 2$, which also coincides with the degenerate limit between Models I and III, we are unable to identify the appropriate wetting law at this time. Instead, we use our solution to the general problem to show how measures of the elastic response vary with the 
relevant system parameters. The strategy is to use the theory to suggest experimental efforts to resolve this dispute, which is of practical importance in moving the field forward.

\section{Appendix: Computation of the constants $C, D$}

The integrals $A_{k}$, defined in eqn (25), can be evaluated by interchanging the order of integration with respect to $\mathrm{d} r \mathrm{~d} t \rightarrow$ $\mathrm{d} t \mathrm{~d} r$ and making use of a Bessel function identity,

$$
J_{0}(s r)=\frac{2}{\pi} \int_{0}^{r} \frac{\cos s t}{\sqrt{r^{2}-t^{2}}} \mathrm{~d} t
$$

which yields

$$
\begin{aligned}
A_{1}(s)=\Delta & \Sigma R \frac{s^{6} \sinh s\left(J_{0}(s R)^{2}+J_{1}(s R)^{2}\right)}{4(1-2 \nu)} \\
A_{2}(s)= & \Delta \Sigma \frac{1}{2} R s^{5}\left(J_{0}(s R)^{2}+J_{1}(s R)^{2}\right) \\
& \times(s \cosh s+(4 \nu-3) \sinh s) .
\end{aligned}
$$

Finally, we apply scalings (15) and simultaneously solve ((20a) and (24)) to give

$$
\begin{aligned}
C \times X(s) /(2(-1+2 \nu))= & 2 \hat{F}_{z}(s)(s \cosh s+(-1+2 \nu) \sinh s) \\
& +\hat{F}_{r}(s)(2 s \sinh s+4(-1+\nu) \cosh s \\
& +r_{\mathrm{sg}}\left(s^{2}(1+\nu) \cosh s\right. \\
& \left.+s\left(-3+\nu+4 \nu^{2}\right) \sinh s\right) \\
& -\Delta r \Lambda s(1+v)(s \cosh s \\
& +(-3+4 \nu) \sinh s)\left(J_{0}(s \Lambda)^{2}\right. \\
& \left.\left.+J_{1}(s \Lambda)^{2}\right)\right) \\
D \times X(s)= & \hat{F}_{z}(s)(2 s \sinh s+4(1-\nu) \cosh \mathrm{s}) \\
+ & \hat{F}_{r}(s)\left(2 s \cosh s+\left(2-4 \nu+s^{2} r_{\mathrm{sg}}(1+\nu)\right.\right. \\
& -\Delta r \Lambda s^{2}(1+\nu)\left(J_{0}(s \Lambda)^{2}\right. \\
& \left.\left.\left.+J_{1}(s \Lambda)^{2}\right)\right) \sinh s\right)
\end{aligned}
$$

where

$$
\begin{aligned}
X(s)= & s^{3}\left(5+2 s^{2}+4 \nu(-3+2 \nu)+(3-4 \nu) \cosh 2 s\right. \\
& +s r_{\mathrm{sg}}\left(-1+v^{2}\right)(2 s+(-3+4 v) \sinh 2 s) \\
& -\Delta r \Lambda s\left(-1+\nu^{2}\right)\left(J_{0}(s \Lambda)^{2}+J_{1}(s \Lambda)^{2}\right) \\
& \times(2 s+(-3+4 \nu) \sinh 2 s)),
\end{aligned}
$$

and $\Delta r \equiv r_{\mathrm{sg}}-r_{\mathrm{ls}}$. The applied forces $\hat{F}$ are given by

$$
\hat{F}_{z}(s)=\sin \alpha\left(\Lambda J_{0}(s \Lambda)-\frac{2}{s} J_{1}(s \Lambda)\right), \hat{F}_{r}(s)=F_{\mathrm{CL}, r} \Lambda J_{1}(s \Lambda),
$$

with the coefficient $F_{\mathrm{CL}, r}$ taken from the models given in Table 1.

\section{Acknowledgements}

This work was supported by the National Science Foundation under grant number DMS-0968258. The authors thank Robert Style and Eric Dufresne for sharing their experimental data.

\section{References}

1 G. Lester, J. Colloid Sci., 1961, 16, 315-326.

2 M. Shanahan and P.-G. De Gennes, C. R. Seances Acad. Sci., Ser. 2, 1986, 302, 517-521.

3 M. E. R. Shanahan, J. Phys. D: Appl. Phys., 1987, $20,945$.

4 B. Roman and J. Bico, J. Phys.: Condens. Matter, 2010, 22, 493101.

5 M. Sokuler, G. K. Auernhammer, M. Roth, C. Liu, E. Bonacurrso and H.-J. Butt, Langmuir, 2009, 26, 1544-1547.

6 J. S. Wexler, T. M. Heard and H. A. Stone, Phys. Rev. Lett., 2014, 112, 066102.

7 T. Young, Philos. Trans. R. Soc. London, 1805, 95, 65-87.

8 A. Dupré, Théorie Méchanique de La Chaleur, Paris, GauthierVillars, Paris, France, 1869, p. 369.

9 A. Marchand, S. Das, J. H. Snoeijer and B. Andreotti, Phys. Rev. Lett., 2012, 109, 236101.

10 J. H. Weijs, J. H. Snoeijer and B. Andreotti, Phys. Rev. E: Stat., Nonlinear, Soft Matter Phys., 2014, 89, 042408.

11 R. Shuttleworth, Proc. Phys. Soc., London, Sect. A, 1950, 63, 444.

12 R. W. Style and E. R. Dufresne, Soft Matter, 2012, 8, 71777184.

13 J. Huang, M. Juszkiewicz, W. H. de Jeu, E. Cerda, T. Emrick, N. Menon and T. P. Russell, Science, 2007, 317, 650-653.

14 D. Vella, M. Adda-Bedia and E. Cerda, Soft Matter, 2010, 6, 5778-5782.

15 B. Davidovitch, R. D. Schroll, D. Vella, M. Adda-Bedia and E. Cerda, Proc. Natl. Acad. Sci. U. S. A., 2011, 108.

16 R. Schroll, M. Adda-Bedia, E. Cerda, J. Huang, N. Menon, T. Russell, K. Toga, D. Vella and B. Davidovitch, Phys. Rev. Lett., 2013, 111, 014301.

17 S. Jung, P. M. Reis, J. James, C. Clanet and J. W. M. Bush, Phys. Fluids, 2009, 21, 091110.

18 E. R. Jerison, Y. Xu, L. A. Wilen and E. R. Dufresne, Phys. Rev. Lett., 2011, 106, 186103.

19 R. W. Style, R. Boltyanskiy, Y. Che, J. Wettlaufer, L. A. Wilen and E. R. Dufresne, Phys. Rev. Lett., 2013, 110, 066103.

20 C. Spandagos, T. B. Goudoulas, P. F. Luckham and O. K. Matar, Langmuir, 2012, 28, 7197-7211.

21 C. Spandagos, T. B. Goudoulas, P. F. Luckham and O. K. Matar, Langmuir, 2012, 28, 8017-8025.

22 K. E. Daniels, S. Mukhopadhyay, P. J. Houseworth and R. P. Behringer, Phys. Rev. Lett., 2007, 99, 124501.

23 J. B. Bostwick and K. E. Daniels, Phys. Rev. E: Stat., Nonlinear, Soft Matter Phys., 2013, 88, 042410.

24 R. W. Style, C. Hyland, R. Boltyanskiy, J. S. Wettlaufer and E. R. Dufresne, Nat. Commun., 2013, 4.

25 L. Tanner, J. Phys. D: Appl. Phys., 1979, 12, 1473.

26 E. van Dussan, Annu. Rev. Fluid Mech., 1979, 11, 371-400.

27 L. Hocking, J. Fluid Mech., 1992, 239, 671-681.

28 T. Kajiya, A. Daerr, T. Narita, L. Royon, F. Lequeux and L. Limat, Soft Matter, 2013, 9, 454-461.

29 T. Kajiya, P. Brunet, A. Daerr, L. Royon, T. Narita, F. Lequeux and L. Limat, Interfacial Phenomena and Heat Transfer, 2013, 1. 
30 A. Leh, H. E. Nguessan, J. Fan, P. Bahadur, R. Tadmor and Y. Zhao, Langmuir, 2012, 28, 5795-5801.

31 L. Chen, E. Bonaccurso and M. E. R. Shanahan, Langmuir, 2013, 29, 1893-1898.

32 B. B. J. Stapelbroek, H. P. Jansen, E. S. Kooij, J. H. Snoeijer and A. Eddi, Soft Matter, 2014, 10, 2641-2648.

33 L. Limat, Eur. Phys. J. E: Soft Matter Biol. Phys., 2012, 35, 1-13.

34 S. Das, A. Marchand, B. Andreotti and J. H. Snoeijer, Phys. Fluids, 2011, 23, 072006.

35 J. H. Weijs, B. Andreotti and J. H. Snoeijer, Soft Matter, 2013, 9, 8494-8503.
36 P. Mott and C. Roland, Phys. Scr., 2013, 87, 055404.

37 J. Mark, Polymer Data Handbook, Oxford University Press, Incorporated, 1999.

38 R. H. Pritchard, P. Lava, D. Debruyne and E. M. Terentjev, Soft Matter, 2013, 9, 6037-6045.

39 R. Soutas-Little, Elasticity, Dover Publications, 1999.

40 I. Sneddon, Mixed Boundary Value Problems in Potential Theory, John Wiley \& Sons, New York,NY, 1966.

41 N. Hoshan, Int. J. Contemp. Math. Sciences, 2009, 4, 16951699. 